\title{
Review of B lifetimes
}

\author{
Kenneth Österberg* on behalf of the LEP, PEP-II, SLC and Tevatron experiments ${ }^{\dagger}$ \\ CERN, CH-1211 Geneva 23, Switzerland \\ E-mail: kenneth.osterberg@cern.ch
}

\begin{abstract}
New precise measurements of $\tau\left(\mathrm{B}^{+}\right), \tau\left(\mathrm{B}_{d}^{0}\right)$ and their ratio have been made by the BaBar, CDF and DELPHI experiments using very different methods to identify the $\mathrm{B}$ species. The BaBar and CDF analysis are based on fully reconstructed $\mathrm{B}$ mesons giving $\tau\left(\mathrm{B}^{+}\right) / \tau\left(\mathrm{B}_{d}^{0}\right)=1.082 \pm 0.026$ (stat.) \pm 0.012 (syst.) and $1.093 \pm 0.066$ (stat.) \pm 0.028 (syst.) respectively. The DELPHI analysis uses a topological reconstruction of the B mesons, where the $\mathrm{B}$ species is determined from the characteristics of the secondary vertex and of leading fragmentation particles, giving a $\tau\left(\mathrm{B}^{+}\right) / \tau\left(\mathrm{B}_{d}^{0}\right)=1.054 \pm 0.017$ (stat.) \pm 0.027 (syst.). These measurements are all consistent with each other and, combined with earlier measurements, a $\tau\left(\mathrm{B}^{+}\right) / \tau\left(\mathrm{B}_{d}^{0}\right)$ average of $1.068 \pm 0.016$ is obtained. This confirms the expectation from theory that the $\tau\left(\mathrm{B}^{+}\right)$should be slightly larger than the $\tau\left(\mathrm{B}_{d}^{0}\right)$.
\end{abstract}

\section{Introduction}

In addition to testing models of B-hadron decays, the knowledge of B-hadron lifetimes is of importance for the determination of the CKM matrix element $V_{c b}$ and as input for time dependent $\mathrm{B}$ decay analysis such as $\mathrm{CP}$ asymmetry measurements and $\mathrm{B}$ meson mixing.

In the spectator model, where the light quark constituent(s) of the B hadron play no role in the decay dynamics and therefore the lifetime depends only on the process $\mathrm{b} \rightarrow \mathrm{cW}^{-}$, all B-hadron species are predicted to have the same lifetime. However nonspectator effects such as quark interference, W exchange and weak annihilation should induce lifetime differences among the different B-hadron species in a similar way to that already experimentally established in the charm hadron system. Due to the relatively high b-quark mass, the lifetime differences are expected to be smaller than in the charm system. Models [1] of $\mathrm{B}$ decays based on expansions in the inverse of the $\mathrm{b}$ quark mass predict the hierarchy, $\tau\left(\Lambda_{b}\right)<\tau\left(\mathrm{B}_{d}^{0}\right) \approx \tau\left(\mathrm{B}_{s}^{0}\right)<\tau\left(\mathrm{B}^{+}\right)$, with differences of the order $5-10 \%$ between the $\mathrm{B}^{+}$and $\mathrm{B}_{d}^{0}$ meson lifetimes. Some assumptions of these models are questioned [2] so precise lifetime measurements of all B-species are clearly needed to clarify the situation.

${ }^{*}$ Speaker.

${ }^{\dagger}$ I want to acknowledge G. Barker (DELPHI and LEP B Lifetime working group), C. Haag (DELPHI), G. Hamel de Monchenault (BaBar) and A. Heiss (CDF) for their contributions to the presentation content. 


\section{2. $\tau\left(\mathrm{B}^{+}\right)$and $\tau\left(\mathrm{B}_{d}^{0}\right)$ measurements in BaBar}

The BaBar measurements are based on data collected in 1999 and 2000 at center-of-mass energies near the $\Upsilon(4 S)$ resonance. The luminosity used in the analysis corresponds to about $23 \mathrm{M} \mathrm{BB}$-pairs. The $\mathrm{B}^{+}$and $\mathrm{B}_{d}^{0}$ mesons are fully reconstructed, whereas an inclusive reconstruction is used for the second $\mathrm{B}$. The asymmetric-energy $\mathrm{e}^{+} \mathrm{e}^{-}$collider PEP-II produces B-meson pairs with a boost of $\beta_{z} \gamma=0.56$ along the beam axis ( $z$ direction). Hence, on average, the two $\mathrm{B}$ decay vertices are separated by $\langle|\Delta z|\rangle=\beta_{z} \gamma \gamma_{B}^{C M S} c \tau \approx 270$ $\mu \mathrm{m}$, where $\gamma_{B}^{C M S}$ is the Lorentz factor of the B meson in the $\Upsilon(4 S)$ rest frame. The difference of the proper decay times of the $\mathrm{B}$ mesons, $\Delta t$, is determined from $\Delta z$, correcting on an event-by-event basis for $\gamma_{B}^{C M S}$. The width of the $\Delta z$ distribution is a combination of lifetime effects and the experimental resolution in $\Delta z$, on average $190 \mu \mathrm{m}$ in this analysis.

The $\mathrm{B}^{+}$meson is reconstructed in its decays to $\mathrm{D}^{(*) 0} \pi^{+}, \mathrm{J} / \psi \mathrm{K}^{+}$and $\psi(2 S) \mathrm{K}^{+}$and the $\mathrm{B}_{d}^{0}$ meson in its decays to $\mathrm{D}^{(*)-} \pi^{+}, \mathrm{D}^{(*)-} \rho^{+}$, $\mathrm{D}^{(*)-} a_{1}^{+}$and $\mathrm{J} / \psi \mathrm{K}^{* 0}$, excluding the $\mathrm{CP}$ asymmetry measurement channel $\mathrm{K}^{* 0} \rightarrow \mathrm{K}_{s}^{0} \pi^{0}$. Various momentum, mass, lepton identification and vertex constraints, described in detail in Ref.[3], are put on the resonances and decays in the chain to ensure the compatibility of the event with one of the above mentioned decay chains. Partially reconstructed B mesons are further rejected requiring the difference of the reconstructed energy and the beam energy $\sqrt{s} / 2$ in the $\Upsilon(4 S)$ rest frame to be compatible. The decay position of the second B meson is reconstructed inclusively from all remaining particles in the event, after removing those of the $\mathrm{B}^{+}$or $\mathrm{B}_{d}^{0}$ candidate. 6064 $\pm 70 \mathrm{~B}_{d}^{0}$ and $6336 \pm 63 \mathrm{~B}^{+}$candidates are found, with a purity of $90 \%$ and a cross-talk of only 1-2 \% between the two samples, after requiring the candidate to have a beam-energy substituted mass, $m_{E S}$, calculated from $\sqrt{s} / 2$ and the reconstructed momentum of the candidate, within $2.5 \sigma$ of the nominal $\mathrm{B}$ mass.

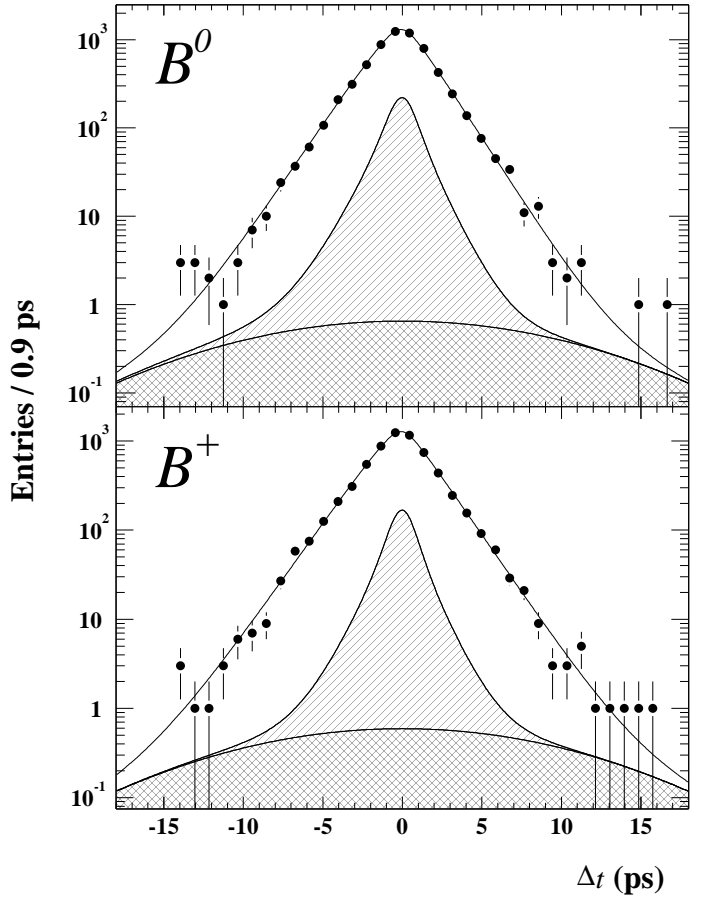

Figure 1: Difference of proper decay times for $\mathrm{B}_{d}^{0}$ (top) and $\mathrm{B}^{+}$(bottom) events with a reconstructed $\mathrm{B}$ mass within $2.5 \sigma$ of the nominal $\mathrm{B}$ mass in the BaBar analysis. The result of the lifetime fit is superimposed on the data. The single-hatched areas corresponds to backgrounds and the cross-hatched to outliers.

The lifetime is extracted from a unbinned maximum likelihood fit to the data extracting simultaneously the lifetime and the resolution function for both signal and background together with the fraction of incorrectly measured events, so-called outliers. The weight of an event in the fit is based on its $m_{E S}$ value. The most significant systematical uncertainties are related to limited $\mathrm{MC}$ statistics, to the parameterization of the resolution functions and to the uncertainty on the outlier resolution function as well as to the background modeling. 
The measured lifetimes together with their statistical and systematical uncertainties are:

$$
\begin{aligned}
\tau\left(\mathrm{B}^{+}\right) & =1.673 \pm 0.032(\text { stat. }) \pm 0.023 \text { (syst.) } \mathrm{ps} \\
\tau\left(\mathrm{B}_{d}^{0}\right) & =1.546 \pm 0.032(\text { stat. }) \pm 0.022(\text { syst. }) \mathrm{ps} \\
\frac{\tau\left(\mathrm{B}^{+}\right)}{\tau\left(\mathrm{B}_{d}^{0}\right)} & =1.082 \pm 0.026(\text { stat. }) \pm 0.012 \text { (syst. }) .
\end{aligned}
$$

\section{Measurement of $\tau\left(\mathrm{B}^{+}\right)$and $\tau\left(\mathrm{B}_{d}^{0}\right)$ at $\mathrm{CDF}$}

An improved lifetime analysis [4] from the CDF collaboration is presented based on their full dimuon and inclusive electron samples from the Tevatron run $\mathrm{I}$. The $\mathrm{B}^{+}$meson is fully reconstructed in its decays to $\mathrm{J} / \psi \mathrm{K}^{+}, \mathrm{J} / \psi \mathrm{K}^{*+}$ and $\psi(2 S) \mathrm{K}^{+}$and the $\mathrm{B}_{d}^{0}$ in its decays to $\mathrm{J} / \psi \mathrm{K}^{* 0}$ and $\mathrm{J} / \psi \mathrm{K}_{s}^{0}$. The lifetime is determined by an unbinned maximum likelihood fit to the proper decay times, as shown in Fig. 2, and the reconstructed B masses of the candidates. The fitted number of signal $\mathrm{B}^{+}$and $\mathrm{B}_{d}^{0}$ events are $1182 \pm$ 43 and $565 \pm 29$ respectively. The fitted purity of the sample is very decay channel dependent ranging between 1.5 and $15 \%$. The main systematical uncertainties are non-gaussian tails of the resolution function and the parameterization of the background. The preliminary lifetimes and their uncertainties are:

$\tau\left(\mathrm{B}^{+}\right)=1.636 \pm 0.058$ (stat.) \pm 0.025 (syst.) ps, $\tau\left(\mathrm{B}_{d}^{0}\right)=1.497 \pm 0.073$ (stat.) \pm 0.032 (syst.) ps, $\frac{\tau\left(\mathrm{B}^{+}\right)}{\tau\left(\mathrm{B}_{d}^{0}\right)}=1.093 \pm 0.066$ (stat.) \pm 0.028 (syst.) .
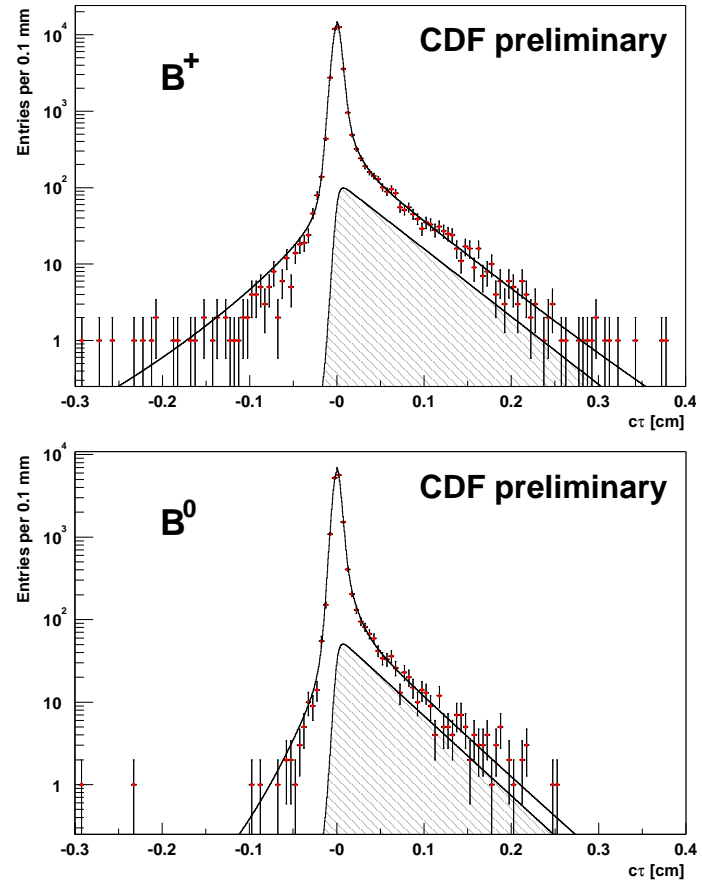

Figure 2: Proper decay time for the $\mathrm{B}^{+}$ (left) and $\mathrm{B}_{d}^{0}$ (right) candidates in the $\mathrm{CDF}$ analysis. The result of the lifetime fit is superimposed on the data. The hatched areas corresponds to the fitted signal.

\section{The DELPHI measurement of $\tau\left(\mathrm{B}^{+}\right)$and $\tau\left(\mathrm{B}_{d}^{0}\right)$}

The DELPHI measurement [5] is based on data collected in 1994 and 1995 at center-of-mass energies near the $\mathrm{Z}^{0}$ resonance. The luminosity used in the analysis corresponds to about $450 \mathrm{k} \mathrm{Z}^{0} \rightarrow \mathrm{b} \overline{\mathrm{b}}$ decays. The $\mathrm{b} \overline{\mathrm{b}}$ decay content in the sample is firstly enhanced using the standard DELPHI b-tagging. The B decay vertex is iteratively reconstructed based on the particle rapidity and particle identification in conjunction with the output of two neural networks. The first network (PSNET) separates primary particles from secondary based on their impact parameters and track rapidity. The second network (BDNET) separates B decay particles from $\mathrm{D}$ decay particles using the impact parameter w.r.t. to the secondary vertex, the momentum of the particle in the B-rest frame and particle identification. 
The BDNET is used to reduce the bias on the reconstructed $B$ decay length due to inclusion of $\mathrm{D}$ decay particles. This bias is further reduced by explicitly removing particles from exclusively reconstructed $\mathrm{D}$ mesons from the vertex and, in case a good lepton candidate is found, to use the lepton-D meson vertex. The decay length resolution is on average about $230 \mu \mathrm{m}$. The B energy is estimated from the particle fourvectors weighted by the PSNET network output. The B energy is further corrected on a hemisphere basis for the missing energy. The $B$ energy resolution is on average about $2.3 \mathrm{GeV}$ for an average $\mathrm{B}$ hadron energy of $32 \mathrm{GeV}$ in onshell $\mathrm{Z}^{0}$ decays. The $\mathrm{B}^{+}$and $\mathrm{B}_{d}^{0}$ mesons are finally identified using the output of a special neural network, seen in Fig. 3, where the decision is based on the PSNET network output weighted secondary vertex charge, the charge and flavour of leading fragmentation particles, the hemisphere energy and several quantities indicating the reconstruction quality as well as the charge correlation with the opposite hemisphere.

At the working point, with a $70 \%$ purity, $57810 \mathrm{~B}^{+}$and 16939 $\mathrm{B}_{d}^{0}$ hemispheres are tagged at a 1 14 and $5 \%$ efficiency respectively. The lifetime is extracted using a binned chi-square fit to the proper decay times as shown by Fig. 4. The most significant systematical uncertainties are related to physics modeling, the event b-tagging and to the B-species neural network performance as well as to the resolution function for the proper de-
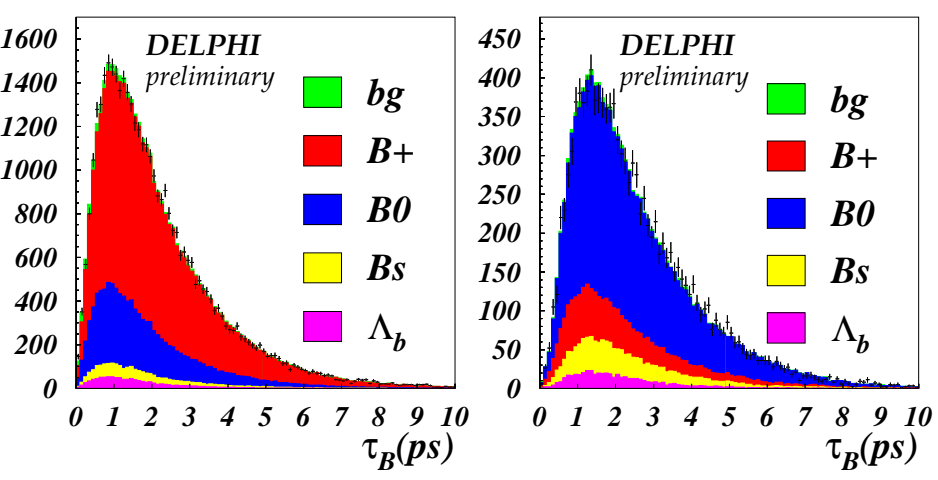

Figure 4: Proper decay time for $\mathrm{B}^{+}$(left)and $\mathrm{B}_{d}^{0}$ (right) candidates in the DELPHI 1994 data. The result of the lifetime fit for the different components is superimposed. cay time and to the uncertainties on the hemisphere reconstruction quality. The extracted preliminary lifetimes and their statistical and systematical uncertainties are: 


$$
\begin{aligned}
\tau\left(\mathrm{B}^{+}\right) & =1.631 \pm 0.012(\text { stat. }) \pm 0.021 \text { (syst. }) \mathrm{ps} \\
\tau\left(\mathrm{B}_{d}^{0}\right) & =1.546 \pm 0.018(\text { stat. }) \pm 0.035(\text { syst. }) \mathrm{ps} \\
\frac{\tau\left(\mathrm{B}^{+}\right)}{\tau\left(\mathrm{B}_{d}^{0}\right)} & =1.054 \pm 0.017 \text { (stat. }) \pm 0.027 \text { (syst. }) .
\end{aligned}
$$

\section{B lifetime averages}

Available measurements of B hadron lifetimes come from the four LEP experiments, BaBar, CDF and SLD experiments. They have been averaged by the LEP B Lifetime working group. The results and the combination method are described in detail in Ref. [6]. The new world averages including the measurements above are:

$$
\begin{aligned}
\tau\left(\mathrm{B}^{+}\right) & =1.647 \pm 0.016 \mathrm{ps} \\
\tau\left(\mathrm{B}_{d}^{0}\right) & =1.546 \pm 0.018 \mathrm{ps} \\
\frac{\tau\left(\mathrm{B}^{+}\right)}{\tau\left(\mathrm{B}_{d}^{0}\right)} & =1.068 \pm 0.016 .
\end{aligned}
$$

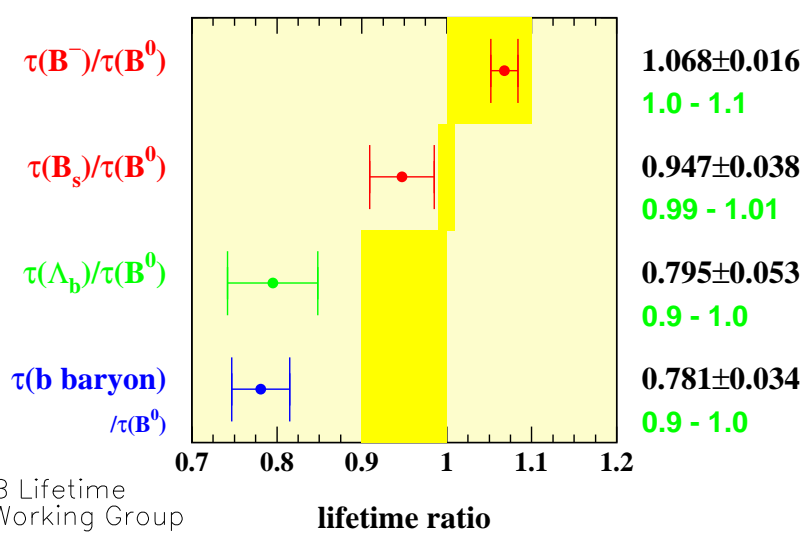

Figure 5: Summary of the new B hadron lifetime averages with the theory predictions of Ref. [1].

No new measurements of $\mathrm{B}_{s}^{0}$ or b-baryon lifetimes are reported. Fig. 5 shows a summary of the current world averages of $\mathrm{B}$ hadron lifetimes and a comparison with predictions from theory. The discrepancy between the expectation and the measurements of b-baryon lifetimes is still present and remains to be understood.

\section{Conclusions}

Several new precise measurements of $\mathrm{B}^{+}$and $\mathrm{B}_{d}^{0}$ meson lifetimes have been presented. It is now experimentally well established, at four standard deviation level, that the $\mathrm{B}^{+}$lifetime is larger than the $\mathrm{B}_{d}^{0}$ lifetime. The $\mathrm{B}$ lifetime hierarchy is correctly predicted by the theory though there is still some discrepancy between the b-baryon lifetime measurements and the predictions. Significant improvements are expected in the near future, for the $\mathrm{B}_{s}^{0}$ and b-baryon lifetimes from the Tevatron and for the $\mathrm{B}^{+}$and $\mathrm{B}_{d}^{0}$ lifetimes from the B-factories.

\section{References}

[1] G. Bellini, I.I. Bigi and P.J. Dornan, Phys. Rep. 289 (1997) 1 and references therein.

[2] M. Neubert and C.T. Sachradja, Nucl. Phys. B 483 (1997) 339.

[3] B. Aubert et al., BaBar Collaboration, to appear in Phys. Rev. Lett., hep-ex/0107019.

[4] A. Heiss et al., CDF Collaboration, CDF/ANAL/BOTTOM/CDFR 5331 (2001) .

[5] G. Barker et al., DELPHI Collaboration, DELPHI 2001-051 CONF 479 (2001) .

[6] J. Alcaraz et al., LEP B Lifetime working group, //home.cern.ch/claires/lepblife.html. 